\title{
On methods of mathematical modeling of drying dispersed materials
}

\author{
Hayvas B. ${ }^{1}$, Dmytruk V. ${ }^{2,1}$, Torskyy A. ${ }^{1,2}$, Dmytruk A. ${ }^{3}$ \\ ${ }^{1}$ Centre of Mathematical Modeling of Pidstryhach IAPMM, NAS of Ukraine \\ 15 Dudayev str., 79005, Lviv, Ukraine \\ ${ }^{2}$ Lviv Polytechnic National University \\ 12 S. Bandera str., 79013, Lviv, Ukraine \\ ${ }^{3}$ Ivan Franko National University of Lviv \\ 1 Universytetska str., 79000, Lviv, Ukraine
}

(Received 20 December 2017)

\begin{abstract}
In this paper there are considered the basic methods of mathematical modeling that are used to describe the processes of convective, convective-thermal and active hydrodynamic drying of dispersed materials, taking into account the structure of individual object of drying. They are described from perspective of continuum mechanics, the theory of mixtures, statistical approaches, which allows taking into account the volume fraction of each phase, the layering of the structure of the object and their changes in the process of phase transitions and chemical transformations.
\end{abstract}

Keywords: drying, dispersed material, suspended state, visco-elastic properties.

2000 MSC: 70G10, 70G70, 76M50, 62E10

UDC: 539.374

DOI: $10.23939 / \mathrm{mmc} 2017.02 .139$

\section{Introduction}

Drying is a complicated process, the optimal flow of which refers to important technological operations, aimed at minimizing the drying time and energy costs, provided that the properties of the dried material would meet the requirements set for them, and in the course of which there is possible an analysis of the changes in time of the average relative moisture of the material and intervention into the kinetics of drying.

\section{Statement of the problem}

Solving important scientific and engineering problems related to the processes of drying of dispersed bodies, in particular, with taking into account the structure of the material, the real mechanisms of heat- and moisture transfer depending on the method of drying, the influence of kinetic, geometric characteristics of the body, controlling parameters of the drying agent, and their influence on the stress-strain state and the stability of the body shape, preserving their quality in the drying process are based on the real macroscopic models whose purpose is to optimize the process of heat and mass transfer.

Such models are constructed on the basis of the general statements of the mechanics of the continuous medium and the thermodynamics of nonequilibrium processes and require effective analytical and numerical methods for solving the corresponding boundary value problems of mathematical physics. The dispersed bodies are multiphase and heterogeneous, therefore, to reflect this, the approaches of the theory of mixtures and the methods of mechanics of multi-velocity systems, combined methods that use capillary models in the simulation of heat and mass transfer processes, while in solving problems of mechanics there are used methods of homogenizing a heterogeneous structure, and as a result there are obtained physical relations for the body as a whole on the basis of certain assumptions about the 
nature of the time and spatial changes of the studied fields. To reflect the influence of the structure of the material, the interaction between the processes of heat and mass transfer inside the body, the influence of the heat and mass exchange between the surfaces of the body and the drying agent on the processes of phase transitions, the effective properties method and stochastic methods are used.

\section{Modeling heterogeneous media}

In multiphase systems there occur effects of the structure and its changes, interphase interaction, in particular capillary effects, phase transitions, rotation and collision of particles [1]. The intermediate position between heterogeneous and homogeneous mixtures is occupied by colloidal mixtures. Solid particles in the dispersed mixture are called dispersed particles, and the carrier continuous phase is called a dispersed phase.

Important are the laws of motion of heterogeneous systems, processes using a stationary granular layer through which the gas mixture is passed; processes with a suspended granular layer under the action of a gas steam; processes in a bed, representing a heterogeneous system, in which the pores are filled with liquid or gas. When considering heterogeneous systems it is assumed that the size of inclusions and inhomogeneities in the mixture is much larger than the molecular-kinetic dimensions, but their size is less than the heterogeneity in which the macroscopic or averaged parameters of the mixture change significantly.

These assumptions allow us to distinguish studies of the behavior of individual inclusions and processes around them. This includes the study of heat transfer, phase transformations, deformations in the particles or in the skeleton of capillary porous bodies, the movement of the mixture in channels, etc. Most products are dried in a dispersed state, which has resulted in the increasing number of usage of different hydrodynamic modes of a suspended layer for their drying. In this case, in each continuum, their macroscopic parameters are determined: velocity, density, pressure, temperature. The results of the microprocesses study are shown in the continuum equations with the help of the averaged parameters of the interaction of the phases.

In homogeneous mixtures (solutions, gas mixtures), the components interact at the molecular or atomic level, the velocities of their relative motion are small and are taken into account only with the determination of the concentrations of the components [2]. The diffusion approximation in the mechanics of mixtures is due to the neglect of the dynamic and inertial effects of diffusion velocities. Relative motions of the components, which are described by diffusion velocities or flows, are determined by a diffusion mechanism, which establishes the dependence on concentration gradients, temperature, and pressure. At the same time, the inertia of the relative motion of the components is neglected [3].

In contrast to homogeneous mixtures, liquids with solid particles (suspensions), water-saturated soils, etc. are described by the multi-velocity model with taking into account the dynamic effects caused by non-matching of component velocities (phases velocities) [4]. In a heterogeneous mixture, each phase occupies only part of the volume, therefore here the proportion of the volume of the mixture is used, and, in addition to the reduced densities, true densities are used. With this, the phases are present in the form of macroscopic relatively to the molecular sizes, and the deformation of each phase which determines its state and reaction is related both to the displacement of the outer boundaries described by the velocity field $v_{i}$, which can differ significantly from the field $v$ of the average-mass velocities of the selected volume, as well as the displacement of the interphase surfaces inside the selected volume of the mixture. Therefore, for each phase, they are considered both an external strain rate tensor

$$
e_{i}^{q p}=\frac{1}{2}\left(\frac{\partial v_{i}^{q}}{\partial x^{p}}+\frac{\partial v_{i}^{p}}{\partial x^{q}}\right)
$$

and the set of tensors

$$
\left(e_{i j}^{* k l}, \quad j=1, \ldots, m, \quad j \neq 1\right),
$$


which take into account the displacement of the substance of the $i$-th phase on the phases interfaces, therefore the true rate of deformation of the phase is determined by the tensor

$$
e_{i}^{* k l}=e_{i}^{k l}+\sum_{\substack{j=1 \\ j \neq i}}^{m} e_{i j}^{* k l} .
$$

The determining $e_{i j}^{* k l}$ is related to the taking into account of the conditions of combined deformation and the movement of phases and conditions that take into account the structure of the constituents of the medium. When the effects of strength do not matter, e.g. in the gas suspended states and in solids at high pressures, the conditions of the combined motion are simpler than in the general case. They are reduced to the equations that determine the volumetric contents of phases $\alpha_{i}$; the equality of pressures of phases; the conditions of incompressibility of one of the phases. If at an arbitrary point of the mixture for each phase it is possible to determine its temperature $T_{i}$, then the state equation, in contrast to the homogeneous case, has the form

$$
\sigma_{i}^{k l}=\sigma_{i}^{k l}\left(\rho_{i}^{0}, e_{i}^{0 m p}, T_{i}, \chi_{i}^{1}, \ldots, \chi_{i}^{r}\right), \quad u_{i}=u_{i}\left(\rho_{i}^{0}, T_{i}, \chi_{i}^{1}, \ldots, \chi_{i}^{r}\right),
$$

that is determined not by the average, but by the true densities $\rho_{i}^{0}$ of the components, $\chi_{i}^{1}, \ldots, \chi_{i}^{r}$ are the auxiliary physico-chemical parameters. The laws that describe the relative motion of the phases are complicated since this motion is determined not only by diffusion processes, but also by the processes of phase interaction as macroscopic systems, for example, the streamline of particles in a gas-suspended state, which should take into account the phase inertia, mass $J_{i j}$, force $P_{i j}$, energy $E_{i j}$ interactions. When the relative motion processes are insignificant in comparison with the average-mass velocity, the dynamic and inertial effects of this motion are small, then a diffusion approximation with some complications due to the presence of the suspended phase can be used to describe the heterogeneous mixtures. An example of a non-inertial diffusion law is Darcy's filtration law. When the inclusions are many times greater than intermolecular sizes, the state equation of the barotropy type

$$
P=P_{i}=P_{i}\left(\rho_{i}^{0}\right)
$$

is used to lock the system of equations.

\section{Modeling the motion of heterogeneous medium with phase transitions}

The exchange of a pulse between $i$-th and $j$-th phases per a unit of time and in a unit of volume is given in the form

$$
P_{j i}=-P_{i j}=R_{j i} v_{j i}, \quad(i, j=1, \ldots, m),
$$

where $R_{j i}$ is the interphase force referred to the unit of volume due to the friction forces, pressure and coupling between phases. The value $v_{j i}$ represents the velocity or momentum of the mass when the state changes $j \rightarrow i$. If in each elemental volume of the mixture each phase is considered to be homogeneous up to the phases separation, when the energy of each component is proportional to its mass, this is equivalent to the fact that the characteristics of the surface layer of a substance are of the order of the radius of molecular interaction $\left(10^{-9} \mathrm{~m}\right)$ [5], which is the limit of the phases differentiation and further are not taken into account.

This requires that all inclusions be many times larger than the thickness of the surface layer. In the energy equation, then, small-scale flows (for example, small-scale chaotic inclusions motions) are not taken into account. The kinetic energy of a mixture with a homogeneous representation is an additive with respect to the phase mass. The introduction of the temperature of each phase is associated with the introduction of the hypothesis of local equilibrium. By the averaging method, there is obtained 
the equations of momentum, the inflow of phases heat and the energy of the pulsating (small-scale) motion [6].

In a biphasic mixture of the solid phase with a liquid or gas [7], the total stress is determined by fictitious stress and pressure from the side of the liquid or gas. Fictitious stresses $\sigma_{f}^{k l}$ are determined by directly measured values $\sigma^{k l}$ and pressure and are interpreted as part of the average stress tensor $\left\langle\sigma_{2}^{k l}\right\rangle_{2}$ in the solid phase or in the skeleton caused by the fluid-independent mechanism of forces transmission through the contacts between the grains. The effects of the strength of the solid phase are manifested in the tensor of fictitious stresses.

In $[8,9]$, the classification of objects of drying is carried out. All solid wet materials are divided into three groups: capillary-porous, colloidal, and capillary-porous colloidal one. Capillary-porous colloids are materials in which the liquid has different forms of binding, which are present in both capillary-porous and colloidal bodies. In particular, in the capillary-porous bodies, the fluid is bound by capillary forces. Being dehydrated, the capillary-porous body become brittle. They compress little and absorb liquid. Capillary forces significantly exceed the force of weight.

Colloid materials include materials with adsorption and osmotically bound moisture. When dried, colloidal bodies are compressed, preserving their elasticity [3]. Being wetted, they absorb the most related by polarity liquids. These are quasi-capillary-porous bodies, the size of which molecules are comparable to the size of the microcapsules.

In [10], the dispersed materials are divided into four groups in order of decreasing the critical pore radius: more than $100 \mathrm{~nm}$; from 100 to 6 ; from 6 to 2; from $2 \mathrm{~nm}$ and less, as well as subgroups that take into account the size of parts of the material, its adhesion-cohesive properties and the duration of drying. The two-phase dispersed mixture, which in the elementary macrovolume contains dispersed particles of the same shape and size, is called monodispersed. If the particles are of different sizes, it is polydispersed. The carrier phase is a spatially interconnected volume (dispersed). An important characteristic of convective drying is the pressure of gases and steam of the drying agent and the material to be dried.

\section{Models of the corpuscular structure}

The dispersion is a thermodynamic characteristic of the system, which determines the area of the phases interface. The degree of dispersion is a degree of fragmentation of the substance of the dispersed phase, reversed to the size of the particles $s=1 / l$, where $l$ is the linear size, which determines the size of the particle. In [11], the method of formation of regular layouts consisting of identical balls is studied. The distance between the balls varies from $\sqrt{3 R}$ that corresponds to the most dense layout, to $\rho_{M}$ that corresponds to the vertical placement of the centres of the layers each over other. The porosity $\varepsilon$ in such layouts varies from 0.2595 to 0.3954 , and the coordination number (the number of contacts of balls with neighbouring balls, which characterizes the stacking of particles) of the densest layout is $N=8$.

In [3], another method of forming regular layouts in the form of layers is studied. The centres of the balls are located at the nodes of the rhombus grids, which are set by the sharp angle $\varpi$ of the rhomb with vertices in the centres of the adjacent layers. The angle $\varpi=60^{\circ}$ corresponds to the densest layout $\varepsilon=0.2595$, and $\varpi=90^{\circ}$ to simple cubic layout $\varepsilon=0.4764$. The coordination number of this layout is equal to $N=12$. If you cut the area around the hollows, so that the plane of the sections will pass through the narrowest sections of the neck of pores, then the intergranular medium is divided into elementary cells - polyhedra. An elementary pore in such a cell represents a hollow bounded by spherical surfaces, which has several necks that connect it with other elementary pores.

In a cubic laying, the hollow of the elementary pore is bounded by 8 balls and is connected by 6 -necks of square form with adjacent hollows. The dimensions of the elementary pores in the basic layers are characterized by the radius of the ball inscribed into the hollow and by the equivalent radius 
$\rho_{\text {neck }}$ inscribed into the neck of the circle. According to Karnaukhov, for basic layouts

$$
\rho_{\text {neck }} \approx 0.47 \frac{\varepsilon}{1-\varepsilon} R
$$

The shape and size of the pores in random layouts of balls are different, but their form is qualitatively similar to the elemental pores in regular layouts. The specific area of particles

$$
S_{y}=S / V_{M}
$$

where $S, V_{M}$ are the area and the volume of particles of the material to be dried.

\section{Drying of natural organic materials}

The process of drying is accompanied by structural changes, physical and chemical transformations, endo- and exothermic reactions. Changes of the isothermal and calorimetric values of the specific heat of evaporation for starch, cellulose caused by the changes in the configuration entropy of the solid components and the entropy of mixing in the interaction of solvent molecules (water) with the macromolecules of high molecular weight substances are noted. However, with the removal of capillary and adsorbed moisture, an increase in the specific heat of evaporation is observed only by $3-5 \%$ and $20 \%$ in comparison to the heat of evaporation of the bulk liquid, respectively.

With low moisture content, individual water molecules are in micropores that are commensurate with the size of the sorbate molecule and interact through hydrogen bonds with the polar functional groups of the sorbent. If pores of larger sizes and are not completely filled, then within them there may be a translational motion of sorbate molecules. As the pore is filled, the mobility decreases. With large moisture content, the material swells, the pore size increases. In them, water molecules are more mobile as filling intermediate and macropores and become close to the mobility of molecules of ordinary water. The process of swelling is similar to the process of water dissolution in a macromolecular substance. With an increase in the moisture content, the water molecules merge and form a continuous phase inside the sorbent and determine the physical characteristics of the sorbate, which are close to the characteristics of the ordinary liquid.

The main indicator characterizing the material as an object of drying is a moisture resistance, which determines the magnitude of the limit residual moisture during dehydration. The living cells possess high fluidity. Physico-chemical properties and reactions to the conditions and limits of dehydration are different. In this regard, the patterns of the effect of residual moisture on the viability of microbial cells, which leads to the final product with low content of viable cells, have not been revealed. When drying biological systems, they are reduced to a dry anabiosis state, in which life processes are suppressed, but the lethal state has not appeared yet and when normal conditions restore, the normal metabolic processes can be recovered. The regulator of the intensity of vital processes of exchange between the cell and the external environment is water $[11,12]$.

Experimental data establish the pattern for vegetative microbial crops of different nature, a crucial loss of viability of microbial cells in the range of moisture content of $50-80 \%$. The effect of residual moisture on the viability of microbial cells is leap-shaped and the main role for lethal result is a sudden destruction of the cell, which can be caused by the rupture of the cell membrane. The value of the critical moisture content corresponding to abrupt change in microbial cell life coincides with the size of the maximal hygroscopic moisture content, and the area of the loss of viability - with the isoterm interval, where moisture is mainly capillary.

For a given relative humidity, the average radius of the capillary is calculated accordind to the Thomson formula

$$
\bar{r}=\frac{2 \rho_{v} \sigma \cos \theta}{\rho_{L} P \ln (1 / \varphi)} .
$$

Mathematical Modeling and Computing, Vol. 4, No. 2, pp. 139-147 (2017) 
Thus, for $\varphi=0.96$ an average radius of the capillary $\bar{r}=10^{-5} \mathrm{~cm}$. In this case, the height of the capillary rise $h=150 \mathrm{~m}$ and the pressure of such a column is about $1.5 \mathrm{MPa}$. If the height of the capillary is less than $h$, then in the capillary there creates a negative pressure that causes deformation of the skeleton of the elastic body (cell membranes). The liquid at negative pressure is in an unstable metastable state. However, the metastable phase is capable of prolonged existence under unchanged thermodynamic conditions, which is associated with a stationary state of an inhaled biological system, an anabiosis. The necessary condition for anabiosis is the presence of a film of metastable liquid around the living cell, which is an element of the structure of the intergranular medium.

In [13], a model of non-stationary processes of a one-component liquid is proposed, taking into account the mutual influence of the vapor pressure, the liquid determined by the contribution of capillary and superficial forces, the temperature on the intensity of mass transfer between phases, thermocapillary flow, the conditions of mechanical and dynamic equilibrium of thin liquid layers on distorted sections of the interface of phases

In [14], the dependence of the coefficient of permeability on the effective pore sizes is obtained, which is based on the differential characteristic of the distribution of the pore material and the Darcy's law for different moisture contents of the material. Determination of the permeability of dry materials is based on the linear dependence of the gas flow through the porous medium from the gas pressure gradient. When removing a liquid from the material having been moistened with a maximum moisture content, first the liquid is released from the largest pores, and then - from smaller ones. In this process, there is a change in the effective porosity of the material and the coefficient of permeability. By maintaining the pressure drop in the filtration constant, it is possible to measure the volume dissipation of air. According to the known values of the thickness of the sample, its filtering surface, and the viscosity of the absorbed liquid, the material's permeability is calculated as a function of the minimum diameter of the open pores. Since the given pressure drop of air compensates the capillary pressure of the liquid in the pores of the material, the minimal diameter of the open pores is determined by the Laplace formula [14]. Under the influence of a leap-shaped increase in the pressure drop, a fluid displacement from smaller pores and a change in the coefficient of permeability are observed. To determine the material's permeability and the value of the minimum diameter of the open pores, it is necessary to measure the moisture content of the sample at each jump of pressure differences $\Delta P$. Experiments show that the effective radius of open pores is strongly influenced by the magnitude of the absorption coefficient.

The quality of the products obtained as a result of drying is determined by the thermal and moisture conditions and the mechanism of heat and mass transfer. The study of diffusion and thermodiffusion processes allows us to know the laws of motion of matter within the body, to find effective ways of controlling the mechanism of mass transfer, to explore new technological schemes of drying processes in order to improve the quality of products. If in the course of drying of moist materials a diffusion of liquid moisture has a place, then water-soluble materials settle on the surface. In the diffusion of vaporous moisture, the water-soluble matter remains within the material.

The method of drying the grain in a suspended state consists in a continuous alternating the processes of grain heating and cooling. When intermittent drying in the process of cooling the grain, the surface layer is more cooled than the internal one. Thermal conductivity in this case helps the moisture conductivity; the flow of heat coincides with the flow of moisture. The evaporation of moisture begins on the surface. Water-soluble nutrients remain in the surface layer, closer to the embryo, which in turn contributes to its growth and helps to increase the seed quality of the grain. During drying of materials, it takes place shrinkage. The material is of good quality, if shrinkage is uniformly in the thickness. With significant differences in moisture content and temperature during the process of drying, in the material a volumetric stress-strain state emerges that exceeds the maximal permissible value. This leads to cracks, or to the complete destruction of the integrity of the material.

Materials can distort or form pores. The surface, which has smaller moisture content, shrinks more, resulting in the sample distort towards the surface with smaller moisture content. In this case, it is 
desirable to act on the mechanism of transfer of moisture so that the mechanism of transfer of moisture on the opposite sides and the rate of moisture transfer from both surfaces are the same. Under the influence of the temperature gradient, the moisture moves inside the material. The flow of moisture directed to the surface is reduced by the amount of moisture flowing due to the flow of thermodiffusion and prevents the movement of moisture from the central layers to the surface. Thermodiffusion reduces the gradient of moisture, the rate of transfer of moisture and the amount of water-soluble substances on the surface of the material. With the change in the direction of the temperature gradient, the physical-chemical and biochemical properties of the material change. In order to preserve the watersoluble substances inside the material, it is necessary that the most intense evaporation occurs inside the material. The temperature gradient increases with increasing air motion velocity. The increase of the temperature gradient during drying of thick materials leads to the emergence of a gradient of moisture content, which leads to stress and crack formation.

In a steam-thermal processing, a saturated vapor is used as a heat-carrier. Heat from the steam-air medium is transferred to the hardening material by convection. A temperature gradient is created in the material that causes the arising of internal stresses. Since the surface temperature is lower than the temperature of the steam-air mixture, the condensate film is formed on the surface and migration of moisture from the surface layers to the central part is observed and the absorption of moisture is observed. The reaction of hydration, which is developing in this time, leads to an excess of material temperature of the medium. As a result of intense evaporation at a significant moisture content, directional pore formation is observed.

When managing the process of moisture transfer, it is important to establish the dependence of the diffusion and thermal diffusion coefficients on the moisture content and temperature, namely the methods of stationary and non-stationary mass transfer. For diatomaceous crumbs, the dependence was obtained for the relative coefficient of thermodiffusion on moisture content $w$ in the form

$$
\delta=a w^{2} c^{-c w},
$$

where $a, c$ are the constants depending on the kind of the material.

The study of the drying of dispersed materials in a suspended state was carried out in [15]. The external heat and mass exchange is considered, which is based on the coupled consideration of the equations of motion and the continuity of the flow of the drying agent and the convective diffusion transfer of the moisture vapour and heat in the moving drying agent. Experimental data on the intensity of external heat and mass transfer are presented in the form of dependence between the similarity criteria derived from the equations and the corresponding uniqueness conditions. The coefficient of mass transfer is included in the Nusselt criterion, whose magnitude is a function of the Reinhold and Prandtl criteria.

Under forced motion of air along the wet surface, the criteria equations for heat exchange and mass transfer are obtained, which are obtained in the experiments on determining the intensity of heat and mass transfer between the drying agent and the material whose surface is maintained in a moist state due to the supply of moisture from the interior zones of the material. Then the moisture is converted into a vapor in the internal zones, the temperature of the external surface increases, and the coefficients of heat and mass transfer can change their values. The change in the coefficient of heat transfer because of the reduced moisture content is taken into account by the ratio of moisture content $w$ to the critical one $w_{k r}$, at which the period of the constant drying rate expires and the surface ceases to be soaked. The approximation formula, which takes into account the contribution to the mass transfer of the frontal and forage portions of the spherical body, is obtained.

Note that the equations of the motion of the drying medium are nonlinear, and for the numerical realization of the velocity alternative function is a diagram of the phase state of the infiltrated dispersed medium constructed in [16], which includes, in addition to the immobile, boiling layer, vertical airtransport also a circulating boiling layer. Expressions are obtained for the calculation of the impor- 
tant characteristic of the flow system (transport velocity). The point on the phase diagram is defined, which determines the value of the velocity of the beginning of the pseudo-liquefaction.

The transfer of heat and mass in a dispersed medium is considered in [17] as occurring in some homogeneous continuum or in several homogeneous continua that coexist at each point of the space, being distracted from the microscopic peculiarities of the transfer near the individual particles. The macroscopic equations of the conservation of heat or mass of the diffusion impurity in a continuous medium containing distributed particles of the dispersed phase are formulated, with neglecting the influence of random fluctuations of the medium and particles on the transfer processes.

\section{Conclusions}

In this paper we have considered the basic methods of mathematical modeling that are used to describe the processes of convective, convective-thermal and active hydrodynamic drying of dispersed systems, taking into account the structure of individual grains, which are based on the diffusion approach and allow taking into account the volume fraction of each phase, the layering of the structure of the object and their changes in the process of phase transitions and chemical transformations. The problems of grain drying are considered, assuming the grain to be a homogeneous structure and taking into account the layering of the grain structure, depending on the location of the grain in the thickness of the layer. The influence of the temperature of the drying agent on the drying process is studied.

[1] Nigmatullin R. I. Fundamentals of mechanics of heterogeneous media. Moscow, Science (1978), (in Russian).

[2] Hersey J. A., Cook P. C. Homogeneity of pharmaceutical dispersed systems. Journal of Pharmacy and Pharmacology. 26 (2), 126-133 (1974).

[3] Gamayunov S. N., Misnikov O. S. Shrinkage phenomena during drying of natural organomineral dispersions. J. Eng. Phys. Thermophys. 71 (2), 234-236 (1998).

[4] Matveenko V.N., Kirsanov E. A. The viscosity and structure of dispersed systems. Moscow University Chemistry Bulletin. 66 (4), 199-228 (2011).

[5] Xue Y. Q., Zhao M. Z., Lai W. P. Size-dependent phase transition temperatures of dispersed systems. Physica B: Condensed Matter. 408 (1), 134-139 (2013).

[6] Mushtaev V. I. The basic theoretical principles of convective drying and the refined method of calculating the drying apparatus. Moscow, MIHM (1971), (in Russian).

[7] Ribitsch V., Jorde C., Schurz J., Jacobasch H. Measuring the zetapotential of fibers, films and granulates. Dispersed Systems. 54, 49-54 (1988).

[8] Mushtaev V. I., Ulyanov V. M. Drying of dispersed materials. Moscow, Chemistry (1988), (in Russian).

[9] Lykov A. V. The theory of drying. Moscow, Energia (1968), (in Russian).

[10] Sazhin B. S. The fundamentals of the technique of drying. Moscow, Chemistry (1984), (in Russian).

[11] Lightfoot E. N. Transport phenomena and living systems: Biomedical applications of momentum and mass transfer. New York, John Wiley (1974).

[12] Tutova E. G., Kuts P. S. Drying of microbiological products. Moscow, Agropromizdat (1987), (in Russian).

[13] Grinchik N. N. Transport processes in porous media, electrolytes and membranes. Minsk, ITMO named after A. V. Lykov of the AN BSSR (1991), (in Russian).

[14] Hayvas B. I., Dmytruk V.A., Dmytruk A.A. Drying of grain in active regimes taking into account its stratified structure. Physical-mathematical modeling and information technologies. 23, 29-42 (2016), (in Ukrainian).

[15] Golik M. G., Delidovich V.N. Scientific fundamentals of grain processing in the stream. Moscow, Kolos (1972), (in Russian).

[16] Dekhtyar R. A., Sikovskii D.F. Heat transfer in a granular layer and moderate Reynolds numbers. Teplofizika Vysokikh Temperatur. 10 (5), 748-755 (2002). 
[17] Egorov G. A. Thermophysical properties of a grain. Information collection. Scientific and technological achievements and best practices in the field of bakery products. Moscow, TSITEhIkhlebprominform (1996), (in Russian).

\title{
Методи математичного моделювання процесів сушіння дисперсних матеріалів
}

\author{
Гайвась Б. ${ }^{1}$, Дмитрук В. ${ }^{2,1}$, Торський А. ${ }^{1,2}$, Дмитрук А. ${ }^{3}$ \\ ${ }^{1}$ Центр математичного моделювання ІППММ ім. Підстригача НАН Украӥни \\ вул. Дудаєва, 15, 79005, Лъвів, Україна \\ ${ }^{2}$ Національний університет «Львівсъка політехніка» \\ вул. С. Бандера, 12, 79013, Львів, Украӥна \\ 3 Львівсъкий начіональний університет імені Івана Франка \\ вул. Університетсъка, 1, 79000, Лъвів, Україна
}

\begin{abstract}
Розглянуто основні методи математичного моделювання, які використовують для опису конвективного, конвективно-теплового та активного гідродинамічного сушіння дисперсних систем з урахуванням структури окремих об'єктів, що грунтується на дифузійному підході і дає змогу враховувати об'ємну частину кожної фази, шаруватість структури об'єкта та зміну їх упродовж фазових переходів та хімічних перетворень.
\end{abstract}

Ключові слова: сушіння, дисперсний матеріал, газозважений стан, в'язко-пружні властивості.

2000 MSC: 70G10, 70G70, 76M50, 62E10

УдК: 539.374 